\title{
TIME-LOCAL DISSIPATIVE FORMULATION AND STABLE NUMERICAL SCHEMES FOR A CLASS OF INTEGRODIFFERENTIAL WAVE EQUATIONS
}

\author{
C. CASENAVE $^{\dagger}$ AND E. MONTSENY ${ }^{\dagger}$
}

\begin{abstract}
We consider integrodifferential equations of the abstract form $\mathbf{H}\left(\partial_{t}\right) \Phi=\mathbf{G}(\nabla) \Phi+f$ where $\mathbf{H}\left(\partial_{t}\right)$ is a diagonal convolution operator and $\mathbf{G}(\nabla)$ is a linear anti self-adjoint differential operator. On the basis of an original approach devoted to integral causal operators, we propose and study a time-local augmented formulation under the form of a Cauchy problem $\partial_{t} \Psi=\mathcal{A} \Psi+\mathcal{B} f$ such that $\Phi=\mathcal{C} \Psi$. We show that under suitable hypothesis on the symbol $\mathbf{H}(p)$, this new formulation is dissipative in the sense of a natural energy functional. We then establish the stability of numerical schemes built from this time-local formulation, thanks to the dissipation of appropriate discrete energies. Finally, the efficiency of these schemes is highlighted by concrete numerical results relating to a model recently proposed for $1 \mathrm{D}$ acoustic waves in porous media.
\end{abstract}

Key words. integrodifferential equation, partial differential equation, convolution operator, diffusive representation, numerical scheme, Cauchy problem, energy functional, stability condition.

\section{AMS subject classifications.}

1. Introduction. In many physical problems where accurate dynamic models are required, the contribution of some underlying and more or less ill-known distributed phenomena cannot be neglected. Although the precise local description of such phenomena often appears excessively complex or even, in many cases, out of scope, their macroscopic dynamic consequences can fortunately most of time be taken into account by means of suitable time-operators of convolution nature which in fact summarize the collective contribution of lots of hidden parameters to the global dynamic behavior of quantities under interest. In that sense, such integrodifferential models therefore conciliate accuracy and simplicity, up to the loss of the so-called time-locality property: in opposite to standard Cauchy problems for which the future is conditioned by the present only, all the past evolution is involved here, via the time-convolution. Last years, various problems relating to integrodifferential models have been studied in many fields. As few examples, we can cite $[2,7,13,16]$ in physics, $[6,10,12]$ in mathematical analysis or numerical simulation, $[1,11]$ in control problems, $[3,9]$ in electrical engineering, [18] in biology, etc.

In the particular context of partial integrodifferential equations, the crucial problem of numerical simulation is in general quite difficult. This is due for one part to the numerical complexity of quadratures of convolution integrals, which generate highly expensive time discretizations, particularly when long memory components are present. Beyond this first heavy shortcoming, the stability of numerical schemes is in general very difficult to get, namely because standard techniques devoted to (ordinary) partial differential equations such as energy dissipation cannot be used for integrodifferential equations. So, the construction of stable numerical schemes remains an important challenge and it can be expected that some specific methods devoted to analysis and approximation of convolution operators should be of great help from this point of view. This is the topic of the present paper.

We consider in the sequel partial integrodifferential equations of the abstract form:

$$
\mathbf{H}\left(\partial_{t}\right) \Phi=\mathbf{G}(\nabla) \Phi+f \text { on }(t, x) \in \mathbb{R}_{t}^{+*} \times \mathbb{R}_{x}^{n},
$$

\footnotetext{
†Laboratoire d'Analyse et d'Architecture des Systèmes, LAAS-CNRS, University of Toulouse,
} Toulouse, France (casenave@laas.fr, emontseny@laas.fr) 
where $\mathbf{H}\left(\partial_{t}\right)$ is an invertible ${ }^{1}$ diagonal convolution operator, and $\mathbf{G}(\nabla)$ is an anti selfajoint linear differential operator. Many propagation phenomena can be modelled following (1.1). As significant examples, we can mention for example electromagnetic waves in dissipative media [13], wave propagation in viscoacoustic media [6], etc. In order to illustrate our results, we will consider in particular a model of $1 \mathrm{D}$ acoustic waves in porous wall proposed in [5]: $\left(H_{1}\left(\partial_{t}\right) u, H_{2}\left(\partial_{t}\right) P\right)^{T}=\left(-\partial_{x} P,-\partial_{x} u\right)^{T}+f$, where $u$ and $P$ stand for the velocity and the pressure of the gas and the symbols $H_{i}$ take the form: $H_{1}(p)=k p+a \sqrt{1+b p}, H_{2}(p)=k^{\prime} p+\frac{c p^{2}}{p+a^{\prime} \sqrt{1+b^{\prime} p}}$.

On the basis of an original approach devoted to integral causal operators presented in $[14,15]$ and successfully applied to various integrodifferential problems, namely in $[1,2,3,9]$, we propose and study a new formulation both equivalent to (1.1) and time-local, written as a Cauchy problem:

$$
\partial_{t} \Psi=\mathcal{A} \Psi+\mathcal{B} f \quad \text { on }(t, x, \xi) \in \mathbb{R}_{t}^{+*} \times \mathbb{R}_{x}^{n} \times \mathbb{R}_{\xi}, \quad \Psi(0, ., .)=0,
$$

in such a way that the solution of (1.1) is expressed $\Phi=\mathcal{C} \Psi$. We show in particular that under natural hypothesis on the symbol $\mathbf{H}(p)$, the formulation (1.2) is dissipative in the sense of an energy functional derived, in some way, from the one of the standard equation $\partial_{t} \Phi=\mathbf{G}(\nabla) \Phi$. Following a convenient method introduced in [14], straightforward dissipative approximate versions of (1.2) are deduced by simple discretization of the auxiliary variable $\xi$. We then study numerical schemes based on classical discretizations relating to the variables $t, x$ and we establish their stability in the sense of adapted energy functionals inherited from the continuous model.

The paper is organized as follows. The section 2 deals with the time-local formulation of (1.1). It begins with a short presentation of the so-called diffusive representation of causal integral operators introduced in [14]; then, the formulation (1.2) is deduced and its dissipativity is established. In section 3, implicit and explicit numerical schemes for (1.2) are stated and studied from the point of view of stability. Finally, the efficiency of these schemes is highlighted in section 4 by means of some numerical simulations.

\section{Time-local formulation of integrodifferential equations.}

2.1. Time local realization of causal convolution operators. In this section, we present a particular case of a methodology called diffusive representation, introduced and developed in [14] in a general framework.

We consider a causal convolution operator denoted by $K\left(\partial_{t}\right)$, that is, for any continuous function $w: \mathbb{R}^{+} \rightarrow \mathbb{R}$,

$$
\left(K\left(\partial_{t}\right) w\right)(t)=\int_{0}^{t} k(t-s) w(s) d s=(k * w)(t)
$$

the function $K=\mathcal{L} k$ (the Laplace transform of $k$ ) is called the symbol of operator $K\left(\partial_{t}\right)$.

Let $w^{t}(s)=\mathbf{1}_{0, t]}(s) w(s)$ and let $w_{t}(s)=w^{t}(t-s)$ the so-called history of $w$. From causality of $K\left(\partial_{t}\right)$, we deduce:

$$
\left(K\left(\partial_{t}\right)\left(w-w^{t}\right)\right)(t)=0 \text { for all } t
$$

\footnotetext{
${ }^{1}$ We implicitly refer to an underlying algebra of causal convolution operators. For example, for a Cauchy problem on $\mathbb{R}_{t}^{+}$with null initial condition, the inverse of $\mathbf{H}\left(\partial_{t}\right)=\partial_{t}$ is $\partial_{t}^{-1}: v \mapsto \int_{0}^{t} v d s$.
} 
then, we have for any continuous function $w$ :

$$
\left(K\left(\partial_{t}\right) w\right)(t)=\left[\mathcal{L}^{-1}(K \mathcal{L} w)\right](t)=\left[\mathcal{L}^{-1}\left(K \mathcal{L} w^{t}\right)\right](t) .
$$

We then define:

$$
\Psi_{w}(t, p):=\mathrm{e}^{p t}\left(\mathcal{L} w^{t}\right)(p)=\left(\mathcal{L} w_{t}\right)(-p) ;
$$

by computing $\partial_{t} \mathcal{L} w_{t}$, Laplace inversion and use of (2.2), it can be shown:

LEMma 2.1. 1. The function $\Psi_{w}$ is solution of the differential equation:

$$
\partial_{t} \Psi(t, p)=p \Psi(t, p)+w, \quad t>0, \Psi(0, p)=0, \quad p \in \mathbb{C}
$$

2. There exists $b_{0} \in \mathbb{R}$ such that:

$$
\forall b \geqslant b_{0},\left(K\left(\partial_{t}\right) w\right)(t)=\frac{1}{2 \mathrm{i} \pi} \int_{b-\mathrm{i} \infty}^{b+\mathrm{i} \infty} K(p) \Psi_{w}(t, p) d p
$$

Proof. 1. From (2.3), we have $\Psi_{w}(t, p):=\mathrm{e}^{p t} \int_{0}^{t} \mathrm{e}^{-p s} w(s) d s$, and so:

$$
\partial_{t} \Psi_{w}(t, p)=p \mathrm{e}^{p t} \int_{0}^{t} \mathrm{e}^{-p s} w(s) d s+\mathrm{e}^{p t} \mathrm{e}^{-p t} w(t) .
$$

2. From (2.2), there exists $b_{0} \in \mathbb{R}$ such that for any $b \geqslant b_{0}$ :

$$
\left(K\left(\partial_{t}\right) w\right)(t)=\frac{1}{2 \mathrm{i} \pi} \int_{b-\mathrm{i} \infty}^{b+\mathrm{i} \infty} \mathrm{e}^{p t} K(p)\left(\mathcal{L} w^{t}\right)(p) d p=\frac{1}{2 \mathrm{i} \pi} \int_{b-\mathrm{i} \infty}^{b+\mathrm{i} \infty} K(p) \Psi_{w}(t, p) d p .
$$

We denote $\Omega$ the holomorphic domain of $K$. Let $\gamma$ a simple arc closed at infinity and included in $\mathbb{C}^{-}=\mathbb{R}^{-}+\mathrm{i} \mathbb{R}$. We denote $\Omega_{\gamma}^{+}$the exterior domain defined by $\gamma$, and $\Omega_{\gamma}^{-}$the complementary of $\overline{\Omega_{\gamma}^{+}}$(see figure 2.1). By use of standard techniques (Cauchy theorem, Jordan lemma), it can be shown:

LEMma 2.2. For $\gamma \subset \Omega$ such that $K$ is holomorphic in $\Omega_{\gamma}^{+}$, if $K(p) \rightarrow 0$ when $p \rightarrow \infty$ in $\Omega_{\gamma}^{+}$, then, for any closed simple arc $\tilde{\gamma}$ in $\Omega_{\gamma}^{+}$such that $\gamma \subset \Omega_{\tilde{\gamma}}^{-}$(see figure 2.1):

$$
\left(K\left(\partial_{t}\right) w\right)(t)=\frac{1}{2 \mathrm{i} \pi} \int_{\tilde{\gamma}} K(p) \Psi_{w}(t, p) d p
$$

We now suppose that $\gamma, \tilde{\gamma}$ are defined by functions of $W_{\text {loc }}^{1, \infty}(\mathbb{R} ; \mathbb{C})$, also denoted $\gamma, \tilde{\gamma}$. From classical techniques, it has been shown in [14] that:

THEOREM 2.3. Under hypothesis of lemma 2.2, if in addition the possible singularities of $K$ on $\gamma$ are simple poles or branching points in the neighborhood of which $|K \circ \gamma|$ is locally integrable, then:

1. with $\tilde{\nu}=\frac{\tilde{\gamma}^{\prime}}{2 \mathrm{i} \pi} K \circ \tilde{\gamma}$ and $\tilde{\psi}(t,)=.\Psi_{w}(t,.) \circ \tilde{\gamma}$ :

$$
\left(K\left(\partial_{t}\right) w\right)(t)=\int_{\mathbb{R}} \tilde{\nu}(\xi) \tilde{\psi}(t, \xi) d \xi
$$

2. if $\tilde{\gamma}_{n} \rightarrow \gamma$ in $W_{\mathrm{loc}}^{1, \infty}$, then $\frac{\tilde{\gamma}_{n}^{\prime}}{2 \mathrm{i} \pi} K \circ \tilde{\gamma}_{n} \rightarrow \nu$ in the sense of measures;

3. $\psi(t,)=.\Psi_{w}(t,.) \circ \gamma$ is the unique solution of the Cauchy problem on $(t, \xi) \in \mathbb{R}^{*+} \times \mathbb{R}$ :

$$
\partial_{t} \psi(t, \xi)=\gamma(\xi) \psi(t, \xi)+w(t), \psi(0, \xi)=0
$$




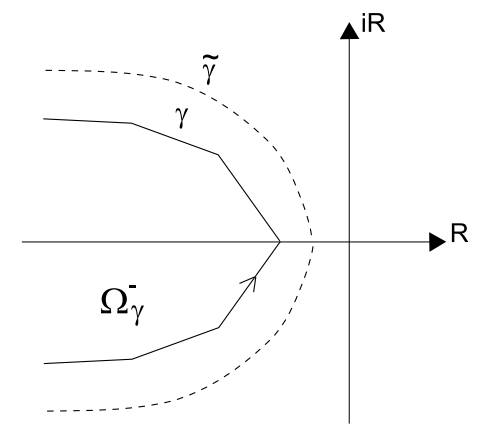

FIG. 2.1. Example of $\gamma$ and $\widetilde{\gamma}$ arcs

and

$$
\left(K\left(\partial_{t}\right) w\right)(t)=\langle\nu, \psi(t, .)\rangle
$$

For convenience, we will indifferently denote in the sequel $\langle\nu, \psi\rangle$ or $\int \nu \psi d \xi$ the duality product between a continuous function $\psi$ and a measure $\nu$ (in particular, for Dirac measures: $\left.\psi(a)=\int \delta_{a} \psi d \xi\right)$.

REMARK 1. In the limit case $\gamma(\xi)=-|\xi|$, we have $\Omega_{\gamma}^{-}=\varnothing$. The above results remain valid and we deduce from symmetry of the problem that there exists a measure $\mu$ such that

$$
\int_{-\infty}^{+\infty} \nu \psi d \xi=\int_{0}^{+\infty} \mu \psi d \xi
$$

This particular case will be useful in practice when $K$ is holomorphic in $\mathbb{C} \backslash \mathbb{R}^{-}$.

Definition 2.4. [14] The measure $\nu$ defined in theorem (2.3) is called the $\gamma$-symbol of operator $K\left(\partial_{t}\right)$.

In many cases, the arc $\gamma$ can be constrained to satisfy a suitable additional condition which makes equation (2.8) of diffusive type [14]. The main advantage of the input-output formulation $(2.8,2.9)$ lies in its time-local nature which allows to use classical methods devoted to Cauchy problems. In particular, stable and efficient schemes for the numerical resolution of (1.1) can be straightforwardly built from discretizations of problem (2.8) following standard techniques. This is the topic of the following sections.

2.2. Application to a class of partial integrodifferential equations. We consider the problem:

$$
\mathbf{H}\left(\partial_{t}\right) \Phi=\mathcal{G} \Phi+f, \quad \text { on } \mathbb{R}_{t}^{+} \times \Omega, \quad \Omega \subset \mathbb{R}_{x}^{m},
$$

where $\Phi=\left(\Phi_{1}, \ldots, \Phi_{M}\right)^{T}$ is the unknown, $\mathbf{H}\left(\partial_{t}\right)$ is an invertible causal convolution operator of the form:

$$
\mathbf{H}\left(\partial_{t}\right)=\left[\begin{array}{lll}
H_{1}\left(\partial_{t}\right) & & \\
& \ddots & \\
& & H_{M}\left(\partial_{t}\right)
\end{array}\right]
$$


and $\mathcal{G}=\mathbf{G}(\nabla)$ is a differential operator supposed to be anti self-adjoint, that is:

$$
\mathcal{G}_{i j}^{*}=-\mathcal{G}_{j i}
$$

where $\mathcal{G}_{i j}^{*}$ is defined by:

$$
\left(\mathcal{G}_{i j} u \mid v\right)_{L^{2}(\Omega)}=\left(u \mid \mathcal{G}_{i j}^{*} v\right)_{L^{2}(\Omega)} \forall u, v \in \mathcal{D}(\Omega)
$$

As usual, suitable boundary conditions associated to $\mathcal{G}$, not expressed here, can complete the model (2.10). The $\gamma_{i}$-symbols $\nu_{i}$ of operators $H_{i}\left(\partial_{t}\right)^{-1}$ are supposed to be positive measures. Note that this property appears as physically realistic in the sense of an energy balance, as it will be highlighted later.

By expressing equation (2.10) under the form $\Phi=\mathbf{H}\left(\partial_{t}\right)^{-1}(\mathcal{G} \Phi+f)$, we formally deduce from results of section 2.1, under suitable hypothesis on $H_{i}^{-1}\left(\partial_{t}\right)$, the following diffusive time-local formulation of (2.10):

$$
\begin{aligned}
\partial_{t} \psi(t, x, \xi) & =\gamma(\xi) \psi(t, x, \xi)+\mathcal{G}\langle\nu, \psi(t, x, .)\rangle+f(t, x), \quad \psi(0, .)=0 \\
\Phi(t, x) & =\langle\nu, \psi(t, x, .)\rangle
\end{aligned}
$$

where $\psi:=\left(\psi_{1}, \ldots, \psi_{M}\right)^{T}, \gamma:=\operatorname{diag}\left(\gamma_{1}, \ldots, \gamma_{M}\right), \nu:=\operatorname{diag}\left(\nu_{1}, \ldots, \nu_{M}\right)$ and $\langle\nu, \psi\rangle:=$ $\left(\left\langle\nu_{1}, \psi_{1}\right\rangle, \ldots,\left\langle\nu_{M}, \psi_{M}\right\rangle\right)^{T}$.

Let us now consider the functional

$$
\psi \longmapsto E_{\psi}=\frac{1}{2} \sum_{i} \iint \nu_{i}\left|\psi_{i}\right|^{2} d \xi d x=\frac{1}{2} \iint \psi^{T} \nu \bar{\psi} d \xi d x ;
$$

thanks to the positivity of $\nu_{i}$, the functional $E_{\psi}$ is positive. We have:

Proposition 2.5. For any $\psi$ solution of (2.13), and at any $t$ such that $f(t, \cdot)=0$, the functional $E_{\psi}$ verifies:

$$
\frac{d E_{\psi}(t)}{d t} \leqslant 0
$$

Proof.

$$
\begin{aligned}
\frac{d E_{\psi}(t)}{d t}= & \frac{1}{2}\left(\iint\left(\partial_{t} \psi\right)^{T} \nu \bar{\psi} d \xi d x+\iint \psi^{T} \nu \overline{\partial_{t} \psi} d \xi d x\right) \\
= & \iint \psi^{T} \nu \operatorname{Re}(\gamma) \bar{\psi} d \xi d x+\frac{1}{2}\left(\int\langle\nu, \psi\rangle^{T} \overline{\mathcal{G}\langle\nu, \psi\rangle} d x+\int(\mathcal{G}\langle\nu, \psi\rangle)^{T} \overline{\langle\nu, \psi\rangle} d x\right) \\
= & \iint \psi^{T} \nu \operatorname{Re}(\gamma) \bar{\psi} d \xi d x \\
& \quad+\frac{1}{2} \sum_{i, j}\left[\left(\mathcal{G}_{i j}\left\langle\nu_{j}, \psi_{j}\right\rangle \mid\left\langle\nu_{i}, \psi_{i}\right\rangle\right)_{L^{2}(\Omega)}+\left(\left\langle\nu_{j}, \psi_{j}\right\rangle \mid \mathcal{G}_{j i}\left\langle\nu_{i}, \psi_{i}\right\rangle\right)_{L^{2}(\Omega)}\right] .
\end{aligned}
$$

Because $\mathcal{G}$ is anti self-adjoint, we then have:

$$
\frac{d E_{\psi}(t)}{d t}=\iint \psi^{T} \nu \operatorname{Re}(\gamma) \bar{\psi} d \xi d x=\sum_{i} \iint \nu_{i} \operatorname{Re}\left(\gamma_{i}\right)\left|\psi_{i}\right|^{2} d \xi \leqslant 0 .
$$


Therefore, the time-local problem (2.13) is dissipative in the sense of the positive functional $E_{\psi}$. At this stage, standard methods of semigroup theory can be investigated to study the well-posedness of this Cauchy problem in the associated energy

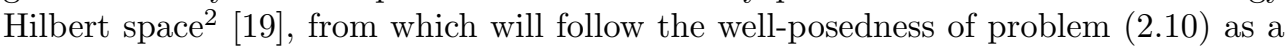
simple consequence.

In practice, the numerical resolution of problems such as (2.10) presents major difficulties due to the non-local nature of $\mathbf{H}\left(\partial_{t}\right)^{-1}$. So, we focus here on the construction and analysis of numerical schemes for (2.13), from which approximate solutions of (2.10) will be directly deduced. We mainly study the stability property, which holds most of the technical difficulties.

3. Numerical schemes for (2.13). First note that in any case, it follows from (2.14) that, in the sense of suitable topologies not specified here, approximations of $\Phi$ solution of (2.10) will be straightforwardly obtained from discrete approximations $\tilde{\psi}$ of $\psi$ solution of (2.13) under the generic form:

$$
\Phi\left(t_{n}, x_{k}\right) \simeq \tilde{\Phi}\left(t_{n}, x_{k}\right)=\sum_{l} \alpha_{l} \tilde{\psi}\left(t_{n}, x_{k}, \xi_{l}\right) .
$$

So, we build and study some numerical schemes for (2.10). A general technique for $\xi$-discretization presented in [14] is first introduced, followed by the statement of fundamental properties of generic $x$-discretizations, inherited from the properties of operator $\mathcal{G}$. Then, we consider different ways of time discretization which define different classes of implicit and explicit schemes.

3.1. $\xi$-discretization [14]. Consider $\mathcal{K}$ a Hilbert space such that $\psi(t, x,.) \in \mathcal{K}$ and $\mathcal{K}_{L}$ a sequence of subspaces of $\mathcal{K}$ of dimension $L$, such that ${\overline{\cup_{L}}}_{\mathcal{K}_{L}} \mathcal{K}=\mathcal{K}$. Given a mesh $\left\{\xi_{l}\right\}_{l=1: L}$, consistent approximations $\widetilde{\psi}_{L} \in \mathcal{K}_{L}$ of $\psi$ are then defined by:

$$
\widetilde{\psi}_{L}(\xi)=\sum_{l=1}^{L} \psi\left(\xi_{l}\right) \Lambda_{l}(\xi),
$$

where $\Lambda_{l}$ are finite element functions belonging to $\mathcal{K}_{L}$ in such a way that:

$$
\left\|\widetilde{\psi}_{L}-\psi\right\|_{\mathcal{K}} \underset{L \rightarrow \infty}{\longrightarrow} 0
$$

We then deduce the finitedimensional approximate state formulation of (2.13):

$$
\partial_{t} \psi\left(t, x, \xi_{l}\right)=\gamma\left(\xi_{l}\right) \psi\left(t, x, \xi_{l}\right)+\mathcal{G} \sum_{j} C_{j} \psi\left(t, x, \xi_{j}\right), \quad l=1: L
$$

where:

$$
C_{l}=\operatorname{diag}\left(c_{l 1}, \ldots, c_{l M}\right), c_{l i}:=\int \nu_{i}(\xi) \Lambda_{l}(\xi) d \xi
$$

Note that, in practice, only a few tens of $\xi_{l}$ are necessary to correctly approximate each operator $H_{i}\left(\partial_{t}\right)^{-1}$. More details on the $\xi$-discretization of diffusive state realizations of convolution operators can be found in [14].

\footnotetext{
${ }^{2} \mathrm{Up}$ to an algebraic quotient in the possible case where supp $\nu \neq \mathbb{R}$, the functional $E_{\psi}$ then defining a seminorm on $\mathcal{D}\left(\mathbb{R}_{x}^{n}\right)$.
} 
In addition, for consistency with positivity of measures $\nu_{i}$, we will suppose:

$$
c_{l i} \geqslant 0
$$

this property, which will play a central role, is satisfied namely if $\Lambda_{l} \geqslant 0$. The energy functional associated to (3.1) is then:

$$
E_{\psi}^{L}(t)=\frac{1}{2} \sum_{i, l} \int c_{l i}\left|\psi_{i}\left(t, x, \xi_{l}\right)\right|^{2} d x=\frac{1}{2} \sum_{l} \int \psi\left(t, x, \xi_{l}\right)^{T} C_{l} \overline{\psi\left(t, x, \xi_{l}\right)} d x
$$

and verifies, in the same way as previously:

$$
\begin{aligned}
\frac{d E_{\psi}^{L}(t)}{d t} & =\sum_{l} \int \psi\left(t, x, \xi_{l}\right)^{T} \operatorname{Re}\left(\gamma\left(\xi_{l}\right)\right) C_{l} \overline{\psi\left(t, x, \xi_{l}\right)} d x \\
& =\sum_{l, i} \int c_{l i} \operatorname{Re}\left(\gamma_{i}\left(\xi_{l}\right)\right)\left|\psi_{i}\left(t, x, \xi_{l}\right)\right|^{2} d x \leqslant 0 .
\end{aligned}
$$

3.2. $x$-discretization. In formulation $(3.1), \mathcal{G}_{i j}$ is a differential operator; it is approximate on a mesh $\left\{x_{k}\right\}_{k=1: K} \subset \mathbb{R}^{m}$ by:

$$
\left(\mathcal{G}_{i j} \Phi\right)\left(x_{q}\right) \simeq \sum_{k=1}^{K} g_{i j}^{q k} \Phi\left(x_{k}\right), \quad \forall q=1: K
$$

where the coefficients $g_{i j}^{q k}$ define the approximation under consideration (for example finite differences [17], finite elements or even more general Galerkin methods up to suitable technical adaptations [4]). By denoting $\widetilde{\Phi}:=\left(\Phi\left(x_{1}\right), \ldots, \Phi\left(x_{K}\right)\right)^{T},(3.3)$ can be written in a more condensed way,:

$$
\left(\left(\mathcal{G}_{i j} \Phi\right)\left(x_{1}\right), \ldots,\left(\mathcal{G}_{i j} \Phi\right)\left(x_{K}\right)\right)^{T} \simeq G_{i j} \widetilde{\Phi}
$$

where we denote $G_{i j}$ the matrix with terms $g_{i j}^{q k}$. In the sequel, for simplicity $\widetilde{\Phi}$ will be denoted $\Phi$.

Because the operator $\mathcal{G}$ is anti self-adjoint, it is natural to consider approximations which preserve this property. So the block matrix $G$ with block elements $G_{i j} \in \mathcal{M}_{K, K}(\mathbb{R})$ must be antisymmetric, that is:

$$
G_{i j}^{T}=-G_{j i}
$$

In the sequel, we will denote $S_{G_{i j}}$ the quantity:

$$
S_{G_{i j}}:=\max \left(\max _{k} \sum_{q}\left|g_{i j}^{q k}\right|, \max _{q} \sum_{k}\left|g_{i j}^{q k}\right|\right) \text {. }
$$

The Euclidian scalar product in $\mathbb{C}^{K}$ and the associated norm will be denoted:

$$
(X \mid Y)=\sum_{k=1}^{K} X_{k} \overline{Y_{k}}, \text { and }\|X\|=\sqrt{\sum_{k=1}^{K}\left|X_{k}\right|^{2}} .
$$


3.3. Stability analysis for an implicit scheme. We propose the following class of time-implicit schemes, based on a Cranck Nicholson time discretization: $(3.5)$

$$
\frac{\psi_{i}^{n+1}\left(\xi_{l}\right)-\psi_{i}^{n}\left(\xi_{l}\right)}{\Delta t}=\gamma_{i}\left(\xi_{l}\right) \frac{\psi_{i}^{n+1}\left(\xi_{l}\right)+\psi_{i}^{n}\left(\xi_{l}\right)}{2}+\sum_{k, j} G_{i k} c_{j k} \frac{\psi_{k}^{n+1}\left(\xi_{j}\right)+\psi_{k}^{n}\left(\xi_{j}\right)}{2}+f_{i}^{n}
$$

where

$$
\psi_{i}^{n}\left(\xi_{l}\right)=\left(\psi_{i}\left(n \Delta t, x_{1}, \xi_{l}\right), \ldots, \psi_{i}\left(n \Delta t, x_{K}, \xi_{l}\right)\right)^{T}
$$

and $f_{i}^{n}=\left(f_{i}\left(n \Delta t, x_{1}\right), \ldots, f_{i}\left(n \Delta t, x_{K}\right)\right)^{T}$. In a more condensed way, (3.5) can be written:

$$
\frac{\psi^{n+1}\left(\xi_{l}\right)-\psi^{n}\left(\xi_{l}\right)}{\Delta t}=\Gamma_{l} \frac{\psi^{n+1}\left(\xi_{l}\right)+\psi^{n}\left(\xi_{l}\right)}{2}+G \sum_{j} Q_{j} \frac{\psi^{n+1}\left(\xi_{j}\right)+\psi^{n}\left(\xi_{j}\right)}{2}+f^{n}
$$

where $\psi^{n}\left(\xi_{l}\right)=\left(\psi_{1}^{n}\left(\xi_{l}\right)^{T}, \ldots, \psi_{M}^{n}\left(\xi_{l}\right)^{T}\right)^{T}, f^{n}=\left(f_{1}^{n T}, \ldots, f_{M}^{n T}\right)^{T}, \Gamma_{l}=\operatorname{diag}\left(\gamma_{i}\left(\xi_{l}\right) I_{K}\right)$, $Q_{j}=\operatorname{diag}\left(c_{j k} I_{K}\right)$ and $G$ is the antisymmetric block matrix defined above.

Let us now consider the quantity:

$$
E^{n}=\sum_{l}\left(\psi^{n}\left(\xi_{l}\right) \mid Q_{l} \psi^{n}\left(\xi_{l}\right)\right)=\sum_{i, l} c_{l i}\left|\psi_{i}^{n}\left(\xi_{l}\right)\right|^{2} .
$$

Note that, thanks to the positivity of coefficients $c_{l i}, E^{n}$ is an energy candidate for (3.6). We have:

TheOREM 3.1. The implicit scheme (3.6) is stable.

Proof.

$$
\begin{aligned}
& E^{n+1}-E^{n}= \\
& =\sum_{l}\left(Q_{l}\left(\psi^{n+1}\left(\xi_{l}\right)+\psi^{n}\left(\xi_{l}\right)\right) \mid \psi^{n+1}\left(\xi_{l}\right)-\psi^{n}\left(\xi_{l}\right)\right)+\sum_{l} 2 \mathrm{i} \operatorname{Im}\left(Q_{l} \psi^{n+1}\left(\xi_{l}\right) \mid \psi^{n}\left(\xi_{l}\right)\right) \\
& =\sum_{l} \frac{\Delta t}{2}\left(Q_{l}\left(\psi^{n+1}\left(\xi_{l}\right)+\psi^{n}\left(\xi_{l}\right)\right) \mid \Gamma_{l}\left(\psi^{n+1}\left(\xi_{l}\right)+\psi^{n}\left(\xi_{l}\right)\right)\right)+\sum_{l} 2 \mathrm{i} \operatorname{Im}\left(Q_{l} \psi^{n+1}\left(\xi_{l}\right) \mid \psi^{n}\left(\xi_{l}\right)\right) \\
& +\frac{\Delta t}{2} \sum_{l, j}\left(Q_{l}\left(\psi^{n+1}\left(\xi_{l}\right)+\psi^{n}\left(\xi_{l}\right)\right) \mid G Q_{j}\left(\psi^{n+1}\left(\xi_{j}\right)+\psi^{n}\left(\xi_{j}\right)\right)\right) .
\end{aligned}
$$

Because $G$ is antisymmetric, we have:

$$
\sum_{l, j}\left(Q_{l}\left(\psi^{n+1}\left(\xi_{l}\right)+\psi^{n}\left(\xi_{l}\right)\right) \mid G Q_{j}\left(\psi^{n+1}\left(\xi_{j}\right)+\psi^{n}\left(\xi_{j}\right)\right)\right)=0,
$$

so:

$$
\begin{aligned}
& E^{n+1}-E^{n}= \\
& =\sum_{l} \frac{\Delta t}{2}\left(Q_{l}\left(\psi^{n+1}\left(\xi_{l}\right)+\psi^{n}\left(\xi_{l}\right)\right) \mid \Gamma_{l}\left(\psi^{n+1}\left(\xi_{l}\right)+\psi^{n}\left(\xi_{l}\right)\right)\right)+\sum_{l} 2 \mathrm{i} \operatorname{Im}\left(Q_{l} \psi^{n+1}\left(\xi_{l}\right) \mid \psi^{n}\left(\xi_{l}\right)\right) \\
& =\frac{\Delta t}{2} \sum_{i, l} \gamma_{i}\left(\xi_{l}\right) c_{l i}\left|\psi_{i}^{n+1}\left(\xi_{l}\right)+\psi_{i}^{n}\left(\xi_{l}\right)\right|^{2}+\sum_{l} 2 \mathrm{i} \operatorname{Im}\left(Q_{l} \psi^{n+1}\left(\xi_{l}\right) \mid \psi^{n}\left(\xi_{l}\right)\right) .
\end{aligned}
$$

As $E^{n+1}-E^{n}$ is real, we have:

$$
E^{n+1}-E^{n}=\frac{\Delta t}{2} \sum_{i, l} c_{l i} \operatorname{Re}\left(\gamma_{i}\left(\xi_{l}\right)\right)\left|\psi_{i}^{n+1}\left(\xi_{l}\right)+\psi_{i}^{n}\left(\xi_{l}\right)\right|^{2} \leqslant 0 .
$$

0 
3.4. Stability analysis for explicit schemes. In this section, we propose a class of two-steps explicit schemes of the form:

$$
\psi_{i}^{n+1}\left(\xi_{l}\right)=a_{l i} \psi_{i}^{n-1}\left(\xi_{l}\right)+b_{l i} \sum_{k} G_{i k} \sum_{j} b_{j k} \psi_{k}^{n}\left(\xi_{j}\right)+b_{l i} f_{i}^{n}
$$

where $a_{l i} \in \mathbb{C},\left|a_{l i}\right|<1$ and $b_{j k} \in \mathbb{R}_{+}^{*}$ are depending both on time approximation and $\gamma_{i}\left(\xi_{l}\right)$ choices, and $G$ is the antisymmetric block matrix associated to operator $\mathcal{G}$.

Let us now study the stability of (3.7). We consider the functional:

$$
E^{n}=\sum_{i, l}\left\|\psi_{i}^{n}\left(\xi_{l}\right)\right\|_{2}^{2}+\operatorname{Re}\left(\psi_{i}^{n+1}\left(\xi_{l}\right) \mid \psi_{i}^{n-1}\left(\xi_{l}\right)\right)
$$

LEMMA 3.2. If

$$
\operatorname{Re}\left(a_{l i}\right)-\frac{b_{l i}}{2} \sum_{k, j} b_{j k} S_{G_{i k}}>0 \quad \forall i, l,
$$

then there exists $K>0$ such that

$$
E^{n} \geqslant K \sum_{i, l}\left\|\psi_{i}^{n}\left(\xi_{l}\right)\right\|^{2}
$$

Proof. We have:

$E^{n}=\sum_{i, l}\left\|\psi_{i}^{n}\left(\xi_{l}\right)\right\|_{2}^{2}+\sum_{i, l} \operatorname{Re}\left(a_{l i}\right)\left\|\psi_{i}^{n-1}\left(\xi_{l}\right)\right\|_{2}^{2}+\sum_{i, l, k, j} b_{l i} b_{j k} \operatorname{Re}\left(G_{i k} \psi_{k}^{n}\left(\xi_{j}\right) \mid \psi_{i}^{n-1}\left(\xi_{l}\right)\right)$.

Moreover, by using the following relation:

$$
\forall \alpha \in \mathbb{R}, \forall u, v \in \mathbb{C}^{K}, \quad \alpha \operatorname{Re}(u \mid v)=\frac{|\alpha|}{2}\left(\|u\|^{2}+\|v\|^{2}-\|u-\operatorname{sign}(\alpha) v\|^{2}\right),
$$

we get:

$$
\begin{aligned}
\operatorname{Re}\left(G_{i k} \psi_{k}^{n}\left(\xi_{j}\right) \mid \psi_{i}^{n-1}\left(\xi_{l}\right)\right) \geqslant \sum_{p, q} & \frac{\left|g_{i k}^{p q}\right|}{2}\left|\psi_{k}^{n}\left(\xi_{j}, x_{q}\right)+\operatorname{sign}\left(g_{i k}^{p q}\right) \psi_{i}^{n-1}\left(\xi_{l}, x_{p}\right)\right|^{2} \\
& -\frac{S_{G_{i k}}}{2}\left\|\psi_{i}^{n-1}\left(\xi_{l}\right)\right\|^{2}-\frac{S_{G_{i k}}}{2}\left\|\psi_{k}^{n}\left(\xi_{j}\right)\right\|^{2}
\end{aligned}
$$

so, as $S_{G_{i k}}=S_{G_{k i}}$ :

$$
\begin{array}{r}
E^{n} \geqslant \sum_{i, l}\left(1-\frac{b_{l i}}{2} \sum_{k, j} b_{j k} S_{G_{i k}}\right)\left\|\psi_{i}^{n}\left(\xi_{l}\right)\right\|^{2}+\sum_{i, l}\left(\operatorname{Re}\left(a_{l i}\right)-\frac{b_{l i}}{2} \sum_{k, j} b_{j k} S_{G_{i k}}\right)\left\|\psi_{i}^{n-1}\left(\xi_{l}\right)\right\|^{2} \\
+\sum_{i, j, k, l, p, q} b_{l i} b_{j k} \frac{\left|g_{i k}^{p q}\right|}{2}\left|\psi_{k}^{n}\left(\xi_{j}, x_{q}\right)+\operatorname{sign}\left(g_{i k}^{p q}\right) \psi_{i}^{n-1}\left(\xi_{l}, x_{p}\right)\right|^{2}
\end{array}
$$

$\square$

REMARK 2. Note that condition (3.8) necessarily implies $\operatorname{Re}\left(a_{l i}\right)>0$; the hypothesis $\left|a_{l i}\right|<1$ is motivated by the term $a_{l i} \psi_{i}^{n-1}\left(\xi_{l}\right)$ of (3.7). 
REMARK 3. Conditions of Lemma 3.2 are necessary conditions that link $\Delta t$ (in $a_{l i}$ and $b_{i k}$ ) and the space discretization step (in $S_{G_{i k}}$ ).

Let us now consider the quantity:

$$
\mathcal{E}^{n}=E^{n}+E^{n-1}
$$

which, under the conditions of lemma 3.2, defines an energy candidate for (3.7). Then, we have the following theorem for stability of the class of explicit schemes (3.7):

THEOREM 3.3. Under the conditions of lemma 3.2 and if, for any $k, j$,

$\left|a_{j k}\right|^{2}+\frac{b_{j k}}{2} \sum_{i, l}\left\|G_{i k}\right\|^{2} b_{l i}\left(\left.|| a_{l i}\right|^{2}+a_{l i}^{2}-\overline{a_{j k}} a_{l i}-1 \mid+b_{l i} \sum_{p, q} b_{q p}\left(\left|a_{l i}-\overline{a_{j k}}\right|+\left|a_{l i}-\overline{a_{q p}}\right|\right)\right) \leqslant 1$

and

$$
\operatorname{Re}\left(a_{j k}\right)\left(\left|a_{j k}\right|^{2}-1\right)+\left.\frac{b_{j k}}{2} \sum_{i, l} b_{l i}|| a_{j k}\right|^{2}+a_{j k}^{2}-\overline{a_{l i}} a_{j k}-1 \mid \leqslant 0
$$

then the scheme (3.7) is stable.

Proof. After computations and reorganization, we have:

$$
\begin{aligned}
& \mathcal{E}^{n+1}-\mathcal{E}^{n}=E^{n+1}-E^{n-1} \\
& =\sum_{i, l}\left|a_{l i}\right|^{2}\left\|\psi_{i}^{n-1}\left(\xi_{l}\right)\right\|^{2}+\sum_{i, l, k, j} b_{l i} b_{j k} \operatorname{Re}\left(a_{l i} \psi_{i}^{n-1}\left(\xi_{l}\right) \mid G_{i k} \psi_{k}^{n}\left(\xi_{j}\right)\right) \\
& +\sum_{i, l, k, j} b_{l i} b_{j k} \operatorname{Re}\left(G_{i k} \psi_{k}^{n}\left(\xi_{j}\right) \mid \psi_{i}^{n+1}\left(\xi_{l}\right)\right)+\sum_{i, l}\left|a_{l i}\right|^{2} \operatorname{Re}\left(\psi_{i}^{n}\left(\xi_{l}\right) \mid \psi_{i}^{n-2}\left(\xi_{l}\right)\right) \\
& +\sum_{i, l, k, j} b_{l i} b_{j k} \operatorname{Re}\left(a_{l i} \psi_{i}^{n}\left(\xi_{l}\right) \mid G_{i k} \psi_{k}^{n-1}\left(\xi_{j}\right)\right)+\sum_{i, l, k, j} b_{l i} b_{j k} \operatorname{Re}\left(G_{i k} \psi_{k}^{n+1}\left(\xi_{j}\right) \mid \psi_{i}^{n}\left(\xi_{l}\right)\right) \\
& -\sum_{i, l}\left(\left\|\psi_{i}^{n-1}\left(\xi_{l}\right)\right\|^{2}+\operatorname{Re}\left(\psi_{i}^{n}\left(\xi_{l}\right) \mid \psi_{i}^{n-2}\left(\xi_{l}\right)\right)\right)
\end{aligned}
$$

As $G_{i k}=-G_{k i}^{T}$, we have:

$$
\begin{aligned}
& \mathcal{E}^{n+1}-\mathcal{E}^{n}=\sum_{i, l}\left(\left|a_{l i}\right|^{2}-1\right)\left\|\psi_{i}^{n-1}\left(\xi_{l}\right)\right\|^{2}+\sum_{i, l}\left(\left|a_{l i}\right|^{2}-1\right) \operatorname{Re}\left(\psi_{i}^{n}\left(\xi_{l}\right) \mid \psi_{i}^{n-2}\left(\xi_{l}\right)\right) \\
& +\sum_{i, l, k, j} b_{l i} b_{j k}\left(a_{l i}-a_{j k}\right) \operatorname{Re}\left(\left(a_{l i}-\overline{a_{j k}}\right) \psi_{i}^{n}\left(\xi_{l}\right) \mid G_{i k} \psi_{k}^{n-1}\left(\xi_{j}\right)\right) \\
& =\sum_{i, l}\left(\left|a_{l i}\right|^{2}-1\right)\left\|\psi_{i}^{n-1}\left(\xi_{l}\right)\right\|^{2}+\sum_{i, l}\left(\left|a_{l i}\right|^{2}-1\right) \operatorname{Re}\left(a_{l i}\right)\left\|\psi_{i}^{n-2}\left(\xi_{l}\right)\right\|^{2} \\
& +\sum_{i, l, k, j} b_{l i} b_{j k} \operatorname{Re}\left(\left(\left|a_{l i}\right|^{2}+a_{l i}^{2}-\overline{a_{j k}} a_{l i}-1\right) \psi_{i}^{n-2}\left(\xi_{l}\right) \mid G_{i k} \psi_{k}^{n-1}\left(\xi_{j}\right)\right) \\
& \quad+\sum_{i, l, k, j, p, q} b_{l i}^{2} b_{j k} b_{q p} \operatorname{Re}\left(\left(a_{l i}-\overline{a_{j k}}\right) G_{i p} \psi_{p}^{n-1}\left(\xi_{q}\right) \mid G_{i k} \psi_{k}^{n-1}\left(\xi_{j}\right)\right)
\end{aligned}
$$

By using (3.9) and the following relation:

$$
\operatorname{Re}(\beta u \mid v)=\frac{1}{2}\left(|\beta|\|v\|_{2}^{2}+|\beta|\|u\|_{2}^{2}-\|\sqrt{\beta} u+\overline{\sqrt{\beta}} v\|_{2}^{2}\right)
$$


and after reorganization, we obtain:

$$
\begin{aligned}
& \mathcal{E}^{n+1}-\mathcal{E}^{n}=\sum_{i, l}\left(\left|a_{l i}\right|^{2}-1\right)\left\|\psi_{i}^{n-1}\left(\xi_{l}\right)\right\|^{2} \\
& +\sum_{i, l}\left(\operatorname{Re}\left(a_{l i}\right)\left(\left|a_{l i}\right|^{2}-1\right)+\left.\frac{b_{l i}}{2} \sum_{k, j} b_{j k}|| a_{l i}\right|^{2}+a_{l i}^{2}-\overline{a_{j k}} a_{l i}-1 \mid\right)\left\|\psi_{i}^{n-2}\left(\xi_{l}\right)\right\|^{2} \\
& -\frac{1}{2} \sum_{i, l, k, j, p, q} b_{l i}^{2} b_{j k} b_{q p} \| \sqrt{a_{l i}-\overline{a_{j k}}} G_{i p} \psi_{p}^{n-1}\left(\xi_{q}\right)+\overline{\sqrt{a_{l i}-\overline{a_{j k}}} G_{i k} \psi_{k}^{n-1}\left(\xi_{j}\right) \|^{2}} \\
& -\frac{1}{2} \sum_{i, l, k, j} b_{l i} b_{j k}\left\|\sqrt{\left|a_{l i}\right|^{2}+a_{l i}^{2}-\overline{a_{j k}} a_{l i}-1} \psi_{i}^{n-2}\left(\xi_{l}\right)+\sqrt{\left|a_{l i}\right|^{2}+a_{l i}^{2}-\overline{a_{j k}} a_{l i}-1} G_{i k} \psi_{k}^{n-1}\left(\xi_{j}\right)\right\|^{2} \\
& +\frac{1}{2} \sum_{i, l, k, j} b_{l i} b_{j k}\left(\left.|| a_{l i}\right|^{2}+a_{l i}^{2}-\overline{a_{j k}} a_{l i}-1 \mid+\sum_{p, q} b_{l i} b_{q p}\left(\left|a_{l i}-\overline{a_{j k}}\right|+\left|a_{l i}-\overline{a_{q p}}\right|\right)\right)\left\|G_{i k} \psi_{k}^{n-1}\left(\xi_{j}\right)\right\|^{2} .
\end{aligned}
$$

By using the property $\left\|G_{i k} \psi_{k}^{n-1}\left(\xi_{j}\right)\right\| \leqslant\left\|G_{i k}\right\|\left\|\psi_{k}^{n-1}\left(\xi_{j}\right)\right\|$, we then get:

$$
\begin{aligned}
& \mathcal{E}^{n+1}-\mathcal{E}^{n} \leqslant \sum_{k, j}\left(\left|a_{j k}\right|^{2}-1\right)+\frac{b_{j k}}{2} \sum_{i, l}\left\|G_{i k}\right\|^{2} b_{l i}\left[\left.|| a_{l i}\right|^{2}+a_{l i}^{2}-\overline{a_{j k}} a_{l i}-1 \mid\right. \\
& \left.\left.\quad+b_{l i} \sum_{p, q} b_{q p}\left(\left|a_{l i}-\overline{a_{j k}}\right|+\left|a_{l i}-\overline{a_{q p}}\right|\right)\right]\right)\left\|\psi_{k}^{n-1}\left(\xi_{j}\right)\right\|^{2} \\
& +\sum_{i, l}\left(\operatorname{Re}\left(a_{l i}\right)\left(\left|a_{l i}\right|^{2}-1\right)+\left.\frac{b_{l i}}{2} \sum_{k, j} b_{j k}|| a_{l i}\right|^{2}+a_{l i}^{2}-\overline{a_{j k}} a_{l i}-1 \mid\right)\left\|\psi_{i}^{n-2}\left(\xi_{l}\right)\right\|^{2} \\
& -\frac{1}{2} \sum_{i, l, k, j} b_{l i} b_{j k}\left\|\sqrt{\left|a_{l i}\right|^{2}+a_{l i}^{2}-\overline{a_{j k}} a_{l i}-1} \psi_{i}^{n-2}\left(\xi_{l}\right)+\sqrt{\left|a_{l i}\right|^{2}+a_{l i}^{2}-\overline{a_{j k}} a_{l i}-1} G_{i k} \psi_{k}^{n-1}\left(\xi_{j}\right)\right\|^{2} \\
& -\frac{1}{2} \sum_{i, l, k, j, p, q} b_{l i}^{2} b_{j k} b_{q p}\left\|\sqrt{a_{l i}-\overline{a_{j k}}} G_{i p} \psi_{p}^{n-1}\left(\xi_{q}\right)+\overline{\sqrt{a_{l i}-\overline{a_{j k}}} G_{i k} \psi_{k}^{n-1}\left(\xi_{j}\right)}\right\|^{2} .
\end{aligned}
$$

So, if for any $k, j$,

$$
\begin{aligned}
& \left|a_{j k}\right|^{2}+\frac{b_{j k}}{2} \sum_{i, l}\left\|G_{i k}\right\|^{2} b_{l i}\left(\left.|| a_{l i}\right|^{2}+a_{l i}^{2}-\overline{a_{j k}} a_{l i}-1 \mid+b_{l i} \sum_{p, q} b_{q p}\left(\left|a_{l i}-\overline{a_{j k}}\right|+\left|a_{l i}-\overline{a_{q p}}\right|\right)\right) \leqslant 1 \\
& \text { and for any } i, l, \quad \operatorname{Re}\left(a_{l i}\right)\left(\left|a_{l i}\right|^{2}-1\right)+\left.\frac{b_{l i}}{2} \sum_{k, j} b_{j k}|| a_{l i}\right|^{2}+a_{l i}^{2}-\overline{a_{j k}} a_{l i}-1 \mid \leqslant 0,
\end{aligned}
$$

then $\mathcal{E}^{n+1} \leqslant \mathcal{E}^{n}$, from which we deduce $E^{n+1} \leqslant E^{n-1}$. Consequently, we have $E^{n} \leqslant \max \left(E^{0}, E^{1}\right)$; from lemma 3.2 , the scheme is stable.

In section 4 , where a concrete application is presented, we will consider two particular explicit scheme of the form (3.7), based on two time discretizations (the first one is rather classical, and the second can be expected to be more precise):

- in the first scheme, the time derivative is approximate by centered finite differences; we then get:

$$
\psi_{i}^{n+1}\left(\xi_{l}\right)=\left(1+2 \Delta t \gamma_{i}\left(\xi_{l}\right)\right) \psi_{i}^{n-1}\left(\xi_{l}\right)+2 \Delta t \sum_{k} G_{i k} \sum_{j} c_{j k} \psi_{k}^{n}\left(\xi_{j}\right)+2 \Delta t f_{i}^{n}
$$


which, after change of variable $\widetilde{\psi}_{i}^{n+1}\left(\xi_{l}\right)=\psi_{i}^{n+1}\left(\xi_{l}\right) \sqrt{\frac{c_{l i}}{2 \Delta t}}$, is rewritten under the form (3.7) with:

$$
a_{l i}=1+2 \Delta t \gamma_{i}\left(\xi_{l}\right) \text { and } b_{j k}=\sqrt{2 \Delta t c_{j k}}
$$

- the second scheme is based on another time discretization described in appendix A, and can be considered in the case where $\gamma_{i}(\xi)$ is real $\left(\gamma_{i}(\xi)=-\xi\right.$ for example). It is written:

$$
\psi_{i}^{n+1}\left(\xi_{l}\right)=e^{\gamma_{i}\left(\xi_{l}\right) 2 \Delta t} \psi_{i}^{n-1}\left(\xi_{l}\right)+\frac{e^{\gamma_{i}\left(\xi_{l}\right) 2 \Delta t}-1}{\gamma_{i}\left(\xi_{l}\right)}\left(\sum_{k} G_{i k} \sum_{j} c_{j k} \psi_{k}^{n}\left(\xi_{j}\right)+f_{i}^{n}\right) ;
$$

after change of variable $\widetilde{\psi}_{i}^{n+1}\left(\xi_{l}\right)=\psi_{i}^{n+1}\left(\xi_{l}\right) \sqrt{\frac{c_{l i} \gamma_{i}\left(\xi_{l}\right)}{e^{\gamma_{i}\left(\xi_{l}\right) 2 \Delta_{t}}-1}},(3.13)$ is rewritten under the form (3.7) with:

$$
a_{l i}=e^{\gamma_{i}\left(\xi_{l}\right) 2 \Delta_{t}} \text { and } b_{j k}=\sqrt{c_{j k} \frac{e^{\gamma_{k}\left(\xi_{j}\right) 2 \Delta_{t}}-1}{\gamma_{k}\left(\xi_{j}\right)}} .
$$

The stability of those particular schemes is obtained as corollary of the general stability theorem 3.3 :

COROLlary 3.4. Under conditions of lemma 3.2, and if $\Delta t$ is small enough, the two schemes (3.7,3.12) and (3.7,3.14) are stable.

Proof. For the first scheme, we have:

$$
a_{l i}=1+2 \Delta t \gamma_{i}\left(\xi_{l}\right) \text { and } b_{j k}=\sqrt{2 \Delta t c_{j k}},
$$

so that, by supposing $\Delta t$ small enough, conditions (3.10) and (3.11) are respectively equivalent to:

$$
1+4 \Delta t \operatorname{Re}\left(\gamma_{k}\left(\xi_{j}\right)\right) \leqslant 1 \text { and } 4 \Delta t \operatorname{Re}\left(\gamma_{k}\left(\xi_{j}\right)\right) \leqslant 0
$$

which are both verified thanks to the property $\operatorname{Re} \gamma \subset \mathbb{R}^{-}$.

For the second scheme, we have:

$$
a_{l i}=e^{\gamma_{i}\left(\xi_{l}\right) 2 \Delta_{t}} \text { and } b_{j k}=\sqrt{c_{j k} \frac{e^{\gamma_{k}\left(\xi_{j}\right) 2 \Delta_{t}}-1}{\gamma_{k}\left(\xi_{j}\right)}}
$$

so if $\Delta t$ is small enough:

$$
a_{l i} \sim 1+2 \Delta t \gamma_{i}\left(\xi_{l}\right) \text { and } b_{j k} \sim \sqrt{2 \Delta t c_{j k}},
$$

and the same analysis as for the first scheme can be made.

\section{Application to a porous wall model.}

4.1. Problem under consideration. In the context of aircraft motors noise reduction in aerospace industry, specific porous wall was proposed in [5] for absorption of a wide part of the energy of incident acoustic waves. The following frequency model of such a material has been established from analysis of harmonic propagating waves: 


$$
\left\{\begin{array} { l } 
{ e \mathrm { i } \omega \rho _ { \mathrm { eff } } ( \mathrm { i } \omega ) \hat { u } + \partial _ { x } \hat { P } = 0 } \\
{ e \mathrm { i } \omega \chi _ { \mathrm { eff } } ( \mathrm { i } \omega ) \hat { P } + \partial _ { x } \hat { u } = 0 }
\end{array} \quad \text { with } \left\{\begin{array}{l}
\rho_{\mathrm{eff}}(\mathrm{i} \omega)=\rho\left(1+a \frac{\sqrt{1+b \mathrm{i} \omega}}{\mathrm{i} \omega}\right) \\
\chi_{\mathrm{eff}}(\mathrm{i} \omega)=\chi\left(1-\beta \frac{\mathrm{i} \omega}{\mathrm{i} \omega+a^{\prime} \sqrt{1+b^{\prime} \mathrm{i} \omega}}\right),
\end{array}\right.\right.
$$

where $\widehat{u}$ and $\widehat{P}$ designate the Fourier transforms of the velocity and the pressure in the porous medium, $e$ denotes the thickness of the porous wall ${ }^{3}, \rho_{\text {eff }}(\mathrm{i} \omega)$ and $\chi_{\text {eff }}(\mathrm{i} \omega)$ are respectively the so-called effective density of Pride et al. [16] and the effective compressibility of Lafarge [8] and $\rho=\rho_{0} \alpha_{\infty}, \chi=\frac{1}{P_{0}}, a=\frac{8 \mu}{\rho_{0} \Lambda^{2}}, a^{\prime}=\frac{8 \mu}{\rho_{0} \Lambda^{\prime 2}}$, $b=\frac{1}{2 a}, b^{\prime}=\frac{1}{2 a^{\prime}}, 0<\beta=\frac{\gamma-1}{\gamma}<1$. The physical parameters $\rho_{0}, P_{0}, \mu, \gamma, \alpha_{\infty}, \Lambda$, $\Lambda^{\prime}$ are respectively the density and pressure at rest, the dynamic viscosity, the specific heat ratio, the tortuosity, the high frequency characteristic length of the viscous incompressible problem and the high frequency characteristic length of the thermal problem. Note that all these parameters are positive by nature.

The aim of this section is to perform temporal simulations of these equations, based on the schemes previously studied. In the time domain, (4.1) can be written (by replacing $p=i \omega$ by $\partial_{t}$ ):

$$
\left[\begin{array}{cc}
H_{1}\left(\partial_{t}\right) & 0 \\
0 & H_{2}\left(\partial_{t}\right)
\end{array}\right]\left(\begin{array}{l}
u \\
P
\end{array}\right)=\left[\begin{array}{cc}
0 & -\partial_{x} \\
-\partial_{x} & 0
\end{array}\right]\left(\begin{array}{l}
u \\
P
\end{array}\right)
$$

with:

$$
H_{1}(p)=e \rho(p+a \sqrt{1+b p}) \text { and } H_{2}(p)=e p \chi\left(1-\beta \frac{p}{p+a^{\prime} \sqrt{1+b^{\prime} p}}\right) .
$$

The analytic continuations of functions $H_{1}(p)^{-1}$ and $H_{2}(p)^{-1}$ are clearly decreasing at infinity and holomorphic in $\mathbb{C} \backslash \mathbb{R}^{-}$. So, from theorem 2.3 , the time-local formulation (2.13) of (4.2) with $\gamma_{i}(\xi)=-|\xi|$ is valid. It takes the form:

$$
\left\{\begin{array}{l}
\partial_{t} \psi(t, x, \xi)=\left[\begin{array}{cc}
-\xi & 0 \\
0 & -\xi
\end{array}\right] \psi(t, x, \xi)+\left[\begin{array}{cc}
0 & -\partial_{x} \\
-\partial_{x} & 0
\end{array}\right]\left(\begin{array}{l}
\left\langle\nu_{1}, \psi_{1}(t, x, .)\right\rangle \\
\left\langle\nu_{2}, \psi_{2}(t, x, .)\right\rangle
\end{array}\right) \\
u=\left\langle\nu_{1}, \psi_{1}(t, x, .)\right\rangle \\
P=\left\langle\nu_{2}, \psi_{2}(t, x, .)\right\rangle
\end{array}\right.
$$

After computations, the $\gamma$-symbol $\nu_{i}$ associated to operator $H_{i}\left(\partial_{t}\right)^{-1}$ are expressed ( $\delta$ denotes de Dirac measure):

$$
\begin{aligned}
& \nu_{1}(\xi)=\frac{a}{\pi e \rho} \frac{\sqrt{b \xi-1}}{\xi^{2}+\frac{a \xi}{2}-a^{2}} \mathbf{1}_{\xi>2 a}+k_{1} \delta\left(\xi-\xi_{1}\right), \\
& \nu_{2}(\xi)=\frac{a^{\prime} \beta}{\pi e \chi} \frac{\sqrt{b^{\prime} \xi-1}}{\xi^{2}(1-\beta)^{2}+\frac{a^{\prime}}{2} \xi-a^{\prime 2}} \mathbf{1}_{\xi>2 a^{\prime}}+\frac{1}{e \chi} \delta(\xi)+k_{2} \delta\left(\xi-\xi_{2}\right),
\end{aligned}
$$

with

$$
\begin{aligned}
& \xi_{1}=\frac{a(\sqrt{17}-1)}{4}>0, \xi_{2}=\frac{a^{\prime}\left(\sqrt{1+16(1-\beta)^{2}}-1\right)}{4(1-\beta)^{2}}>0, \\
& k_{1}=\frac{\sqrt{17}-1}{e \rho \sqrt{17}}>0, \text { and } k_{2}=\frac{\beta\left(\sqrt{1+16(1-\beta)^{2}}-1\right)}{e \chi(1-\beta) \sqrt{1+16(1-\beta)^{2}}}>0 .
\end{aligned}
$$

\footnotetext{
${ }^{3}$ In the model, the unit of length for $x$ is $e$, so $\left.x \in\right] 0,1[$.
} 
For the $\xi$-discretization, we consider the classical interpolation functions:

$$
\Lambda_{l}(\xi)=\frac{\xi-\xi_{l-1}}{\xi_{l}-\xi_{l-1}} \mathbf{1}_{\left[\xi_{l-1}, \xi_{l}\right]}(\xi)+\frac{\xi_{l+1}-\xi}{\xi_{l+1}-\xi_{l}} \mathbf{1}_{] \xi_{l}, \xi_{l+1}\right]}(\xi)
$$

and coefficients $c_{l i}$ are computed by simple quadrature of $\int \nu_{i}(\xi) \Lambda_{l}(\xi) d \xi$.

4.2. Numerical schemes. In this example, $\mathcal{G}=\left[\begin{array}{cc}0 & -\partial_{x} \\ -\partial_{x} & 0\end{array}\right]$. We use centered finite differences to approximate the derivative operator $\partial_{x}$, so the matrix of $x$-discretization $G$ is given by:

$$
G=\left[\begin{array}{cc}
0 & G_{12} \\
G_{21} & 0
\end{array}\right] \text { with } G_{12}=G_{21}=\frac{1}{2 \Delta x}\left[\begin{array}{ccccc}
0 & -1 & & & \\
1 & 0 & -1 & & \\
& \ddots & \ddots & \ddots & \\
& & 1 & 0 & -1 \\
& & & 1 & 0
\end{array}\right] \text {. }
$$

Note that this matrix is antisymmetric, so that the schemes studied in section 3 can be used. Then, we consider:

- the implicit scheme:

$$
\left\{\begin{array}{l}
\frac{\psi_{1}^{n+1}\left(\xi_{l}\right)-\psi_{1}^{n}\left(\xi_{l}\right)}{\Delta t}=-\xi_{l} \frac{\psi_{1}^{n+1}\left(\xi_{l}\right)+\psi_{1}^{n}\left(\xi_{l}\right)}{2}+G_{12} \sum_{j} c_{j 2} \frac{\psi_{2}^{n+1}\left(\xi_{j}\right)+\psi_{2}^{n}\left(\xi_{j}\right)}{2}+f_{1}^{n} \\
\frac{\psi_{2}^{n+1}\left(\xi_{l}\right)-\psi_{2}^{n}\left(\xi_{l}\right)}{\Delta t}=-\xi_{l} \frac{\psi_{2}^{n+1}\left(\xi_{l}\right)+\psi_{2}^{n}\left(\xi_{l}\right)}{2}+G_{21} \sum_{j} c_{j 1} \frac{\psi_{1}^{n+1}\left(\xi_{j}\right)+\psi_{1}^{n}\left(\xi_{j}\right)}{2}+f_{2}^{n},
\end{array}\right.
$$

- the two particular explicit schemes of the form:

$$
\left\{\begin{array}{l}
\psi_{1}^{n+1}\left(\xi_{l}\right)=a_{l 1} \psi_{1}^{n-1}\left(\xi_{l}\right)+b_{l 1} G_{21} \sum_{j} b_{j 2} \psi_{2}^{n}\left(\xi_{j}\right)+b_{l 1} f_{1}^{n} \\
\psi_{2}^{n+1}\left(\xi_{l}\right)=a_{l 2} \psi_{2}^{n-1}\left(\xi_{l}\right)+b_{l 2} G_{12} \sum_{j} b_{j 1} \psi_{1}^{n}\left(\xi_{j}\right)+b_{l 2} f_{2}^{n}
\end{array}\right.
$$

respectively obtained with:

$$
a_{l i}=1-2 \Delta t \xi_{l}, b_{j k}=\sqrt{2 \Delta t c_{j k}} \quad \text { and } \quad a_{l i}=e^{-\xi_{l} 2 \Delta_{t}}, b_{j k}=\sqrt{c_{j k} \frac{e^{-\xi_{j} 2 \Delta_{t}}-1}{-\xi_{j}}} .
$$

4.3. Physical interpretation of stability conditions. Obviously, to be able to correctly simulate wave propagation phenomena, explicit schemes necessarily have a numerical influence velocity at least equal to the maximal velocity of wave fronts in the medium under consideration. When this is not the case, a consistent explicit scheme cannot be convergent and is therefore unstable. So, it can be expected that the stability conditions of section 3.4 applied to (4.5) can be in some way interpreted in terms of high frequency wave velocity. More precisely: is the sufficient stability condition for (4.5) "optimal" in the sense that it is close to the necessary condition mentioned above? This is studied in the present section.

Let us compute the expression of the high frequency wave velocity of model (4.2), denoted by $c$. We have:

$$
\left\{\begin{array}{l}
u=-H_{1}\left(\partial_{t}\right)^{-1} \partial_{x} P \\
P=-H_{2}\left(\partial_{t}\right)^{-1} \partial_{x} u,
\end{array}\right.
$$


so we get $u=H_{1}\left(\partial_{t}\right)^{-1} H_{2}\left(\partial_{t}\right)^{-1} \partial_{x}^{2} u$. Moreover [14]:

$$
H_{i}(\mathrm{i} \omega)^{-1}=\int \frac{\nu_{i}(\xi)}{\mathrm{i} \omega+\xi} d \xi=\frac{1}{\mathrm{i} \omega} \int \frac{\nu_{i}(\xi)}{1+\frac{\xi}{\mathrm{i} \omega}} d \xi, \quad i=1,2 .
$$

So, when $\omega \rightarrow+\infty$ :

$$
H_{i}(\mathrm{i} \omega)^{-1} \sim \frac{1}{\mathrm{i} \omega} \int \nu_{i}(\xi) d \xi
$$

The equation $u=H_{1}\left(\partial_{t}\right)^{-1} H_{2}\left(\partial_{t}\right)^{-1} \partial_{x}^{2} u$ therefore behaves at high frequency as equation $\partial_{t}^{2} u=c^{2} \partial_{x}^{2} u$, with

$$
c=\sqrt{\int \nu_{1}(\xi) d \xi \int \nu_{2}(\xi) d \xi}
$$

Similarly, we denote by $c_{d}$ the high frequency wave velocity of the continuous model obtained after $\xi$-discretization of $(4.2)$, in which $H_{i}(\mathrm{i} \omega)$ is replaced by its approximation $\widetilde{H}_{i}(\mathrm{i} \omega)[14]$ :

$$
\widetilde{H}_{i}(\mathrm{i} \omega)^{-1}=\sum_{j} \frac{c_{j i}}{\mathrm{i} \omega+\xi_{j}}=\frac{1}{\mathrm{i} \omega} \sum_{j} \frac{c_{j i}}{1+\frac{\xi_{j}}{\mathrm{i} \omega}}, \quad i=1,2
$$

We have, when $|\omega| \rightarrow+\infty$ :

$$
\widetilde{H}_{i}(\mathrm{i} \omega)^{-1} \sim \frac{1}{\mathrm{i} \omega} \sum_{j} c_{j i}
$$

which leads to a high frequency behavior of the form $\partial_{t}^{2} u=c_{d}^{2} \partial_{x}^{2} u$ with

$$
c_{d}=\sqrt{\sum_{j} c_{j 1} \sum_{j} c_{j 2}}
$$

Thanks to the expression of $c_{l i}$, we then have, if $\widetilde{H}_{i}^{-1}$ is sufficiently close to $H_{i}^{-1}$ :

$$
c_{d} \simeq c .
$$

Moreover, $S_{G_{12}}=S_{G_{21}}=\frac{1}{\Delta x}$, so the stability conditions of section 3.4 applied to (4.5) are:

$\Delta t$ small enough and

$$
\forall(i, k) \in\{(1,2),(2,1)\}, \quad \forall l=1: L, \quad a_{l i}-\frac{b_{l i}}{2 \Delta x} \sum_{j} b_{j k}>0 .
$$

For the first explicit scheme, (4.9) is expressed:

$$
\frac{\Delta x}{\Delta t}>v_{d}:=\max _{(i, k)} \max _{l} \frac{\sqrt{c_{l i}}}{1-2 \Delta t \xi_{l}} \sum_{j} \sqrt{c_{j k}},
$$

where $\frac{\Delta x}{\Delta t}$ is the numerical influence velocity of the scheme. For the second explicit scheme, the order one approximation leads to the same condition. Then, we have the following result: 
Proposition 4.1. $v_{d} \geqslant c_{d}$.

Proof. Without loss of generality, we can consider that:

$$
v_{d}=\max _{(i, k)} \max _{l} \frac{\sqrt{c_{l i}}}{1-2 \Delta t \xi_{l}} \sum_{j} \sqrt{c_{j k}}=\max _{l} \frac{\sqrt{c_{l 1}}}{1-2 \Delta t \xi_{l}} \sum_{j} \sqrt{c_{j 2}} .
$$

So we have:

$$
\begin{aligned}
c_{d}^{2}=\sum_{j} c_{j 1} \sum_{j} c_{j 2} & \leqslant \max _{l} \sqrt{c_{l 1}} \sum_{j} \sqrt{c_{j 1}} \max _{l} \sqrt{c_{l 2}} \sum_{j} \sqrt{c_{j 2}} \\
& \leqslant \max _{l} \frac{\sqrt{c_{l 1}}}{1-2 \Delta t \xi_{l}} \sum_{j} \sqrt{c_{j 2}} \max _{l} \frac{\sqrt{c_{l 2}}}{1-2 \Delta t \xi_{l}} \sum_{j} \sqrt{c_{j 1}} \\
& \leqslant\left(\max _{l} \frac{\sqrt{c_{l 1}}}{1-2 \Delta t \xi_{l}}\right)^{2}\left(\sum_{j} \sqrt{c_{j 2}}\right)^{2}=v_{d}^{2} .
\end{aligned}
$$

$\square$

As expected, we deduce from (4.10) and proposition 4.1 that the numerical influence velocity of the scheme necessarily satisfies:

$$
\frac{\Delta x}{\Delta t}>c_{d}
$$

The sufficient stability condition (4.10) is of course not necessary. However, in numerical results of paragraph 4.4, the gap between this condition and the instability of the scheme is small: then, this condition is quasi optimal in this case.

REMARK 4. The numerical velocity $v_{d}$ of (4.5) could also be compared to the one of a (theoretical) scheme in which the variable $\xi$ remains continuous, by considering the continuous equivalent of the quantity $v_{d}=\max _{(i, k)} \max _{l} \frac{\sqrt{c_{l i}}}{1-2 \Delta t \xi_{l}} \sum_{j} \sqrt{c_{j k}}$ in (4.10). Namely, by supposing by simplicity that $\nu_{i}$ are positive and continuous functions $^{4}$ with bounded support, that $\Delta_{\xi}=\xi_{l+1}-\xi_{l}$ is constant and with $\Lambda_{l}=\mathbf{1}_{\left[\xi_{l}, \xi_{l+1}\right]}$, there exists $\nu_{l i}^{\prime} \in\left[\nu_{i}\left(\xi_{l}\right), \nu_{i}\left(\xi_{l+1}\right)\right]$ such that: $c_{l i}=\int_{\xi_{l}}^{\xi_{l+1}} \nu_{i}(\xi) d \xi=\nu_{l i}^{\prime} \Delta \xi ;$ so,

$$
\sqrt{c_{l i}} \sum_{j} \sqrt{c_{j k}}=\sqrt{\nu_{l i}^{\prime}} \sum_{j} \sqrt{\nu_{j k}^{\prime}} \Delta \xi \simeq \sqrt{\nu_{l i}^{\prime}} \int \sqrt{\nu_{k}(\xi)} d \xi
$$

and therefore, with $\Delta t$ such that $1-2 \Delta t \xi \geqslant 0$ for any $\xi \in \operatorname{supp} \nu_{1} \cup \operatorname{supp} \nu_{2}$ :

$$
v_{d} \simeq \max _{(i, k)} \max _{l} \frac{\sqrt{\nu_{l i}^{\prime}}}{1-2 \Delta t \xi_{l}} \int \sqrt{\nu_{k}(\xi)} d \xi \leqslant v:=\max _{(i, k)} \sup _{\xi} \frac{\sqrt{\nu_{i}(\xi)}}{1-2 \Delta t \xi} \int \sqrt{\nu_{k}} d \xi .
$$

Then, similarly to proposition 4.1, it can be easily shown that $v>c$. Note however that besides the boundness of supp $\nu_{i}$ which is a quite unrealistic hypothesis, this estimation can be in some cases excessively pessimist.

4.4. Numerical results. We give in this section some numerical results obtained with the explicit schemes. The values of parameters are [5]:

$$
\begin{aligned}
& \Lambda=\Lambda^{\prime}=0.110^{-3} \mathrm{~m}, \rho_{0}=1.2 \mathrm{~kg} \cdot \mathrm{m}^{-3}, P_{0}=10^{5} \mathrm{~Pa} \\
& \mu=1.810^{-5} \mathrm{~kg} \cdot \mathrm{m}^{-1} \cdot \mathrm{s}^{-1}, \gamma=1.4, \alpha_{\infty}=1.3, e=510^{-2} \mathrm{~m} .
\end{aligned}
$$

The frequency responses of the approximations of $H_{i}\left(\partial_{t}\right)^{-1}$ obtained with (4.7) are given in figure 4.1. Only 15 (resp. 20) $\xi_{l}$ are used to approximate $H_{1}\left(\partial_{t}\right)^{-1}$ (resp. $H_{2}\left(\partial_{t}\right)^{-1}$ ) on a range of 6 decades with a good accuracy.

\footnotetext{
${ }^{4}$ Dirac and $L_{\text {loc }}^{1}$ components could be similarly treated up to suitable technical adaptations.
} 

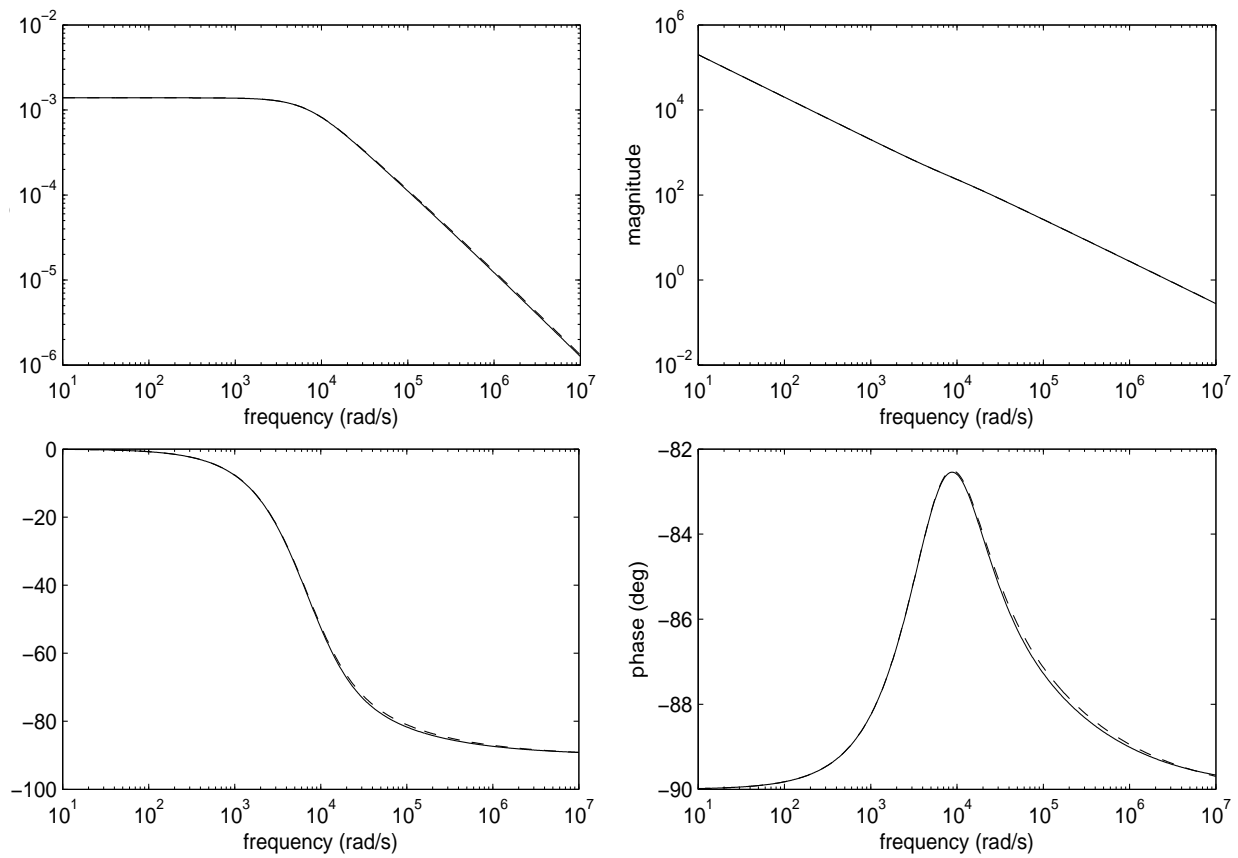

FIG. 4.1. Exact (-) and approximate (- - ) frequency responses of operators $H_{1}\left(\partial_{t}\right)^{-1}$ (at left) and $H_{2}\left(\partial_{t}\right)^{-1}$ (at right)

For illustration, the evolution of $P$ obtained from simulation with explicit schemes is shown in figure 4.2 (the two curves are superposed); the $x$-domain of (4.2) is $\Omega=$ ] $0,1[$ and boundary conditions are:

$$
P(t, 0)=(1-\cos (2 \pi f t)) \mathbf{1}_{\left[0, \frac{1}{f}\right]}(t), \quad u(t, 1)=0,
$$

with $f=5 k H z$.We can clearly observe the dissipation and dispersion due to operator $\mathbf{H}\left(\partial_{t}\right)$.

In figure 4.3 we can see at a particular time, the functions $\psi_{1}$ which are involved in the synthesis of $u$. 

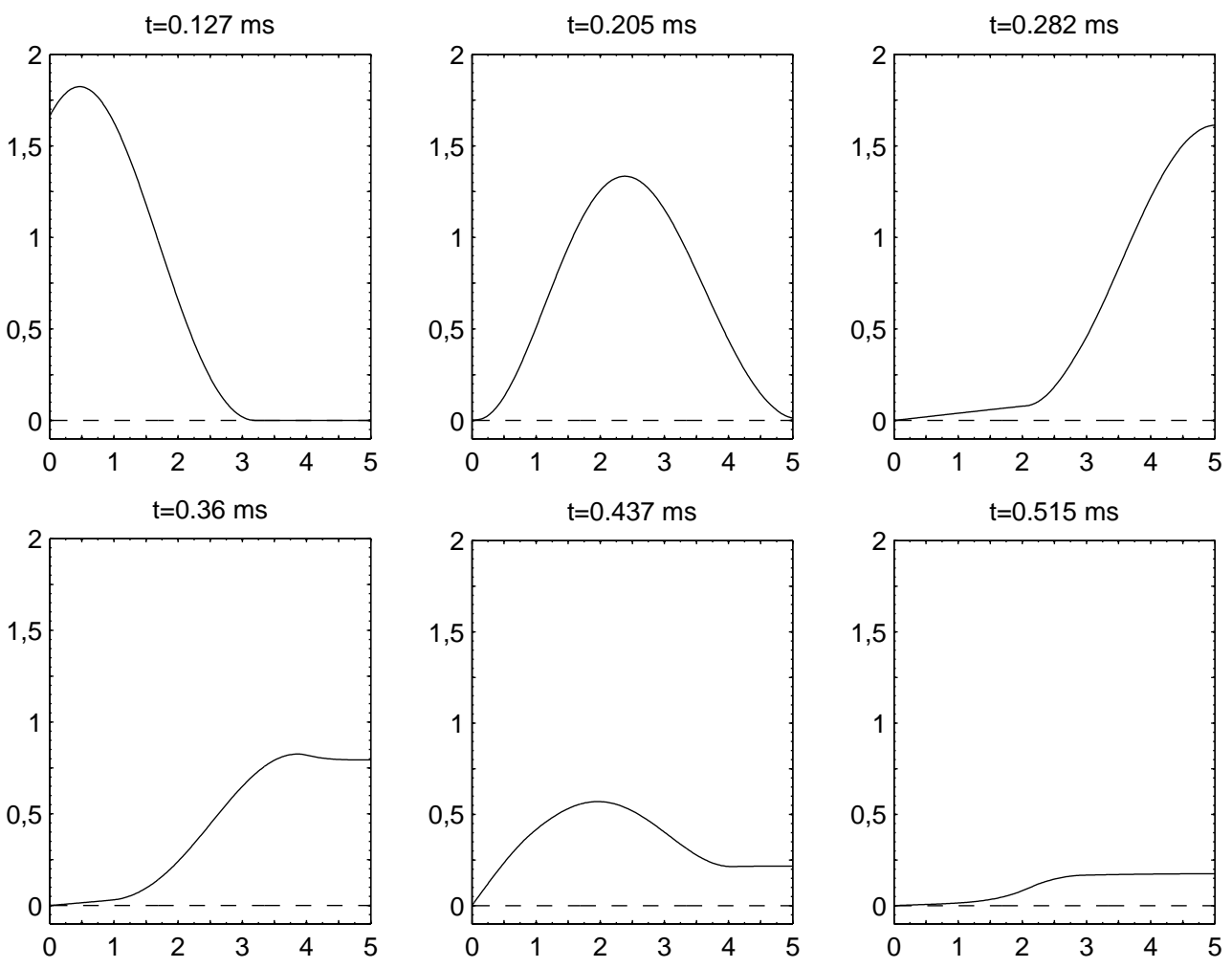

FIG. 4.2. Evolution of $\widetilde{P}=\sum_{l} b_{l 2} \psi_{2}\left(\xi_{l}\right)$ (N.B: the unit of length for the $x$-axis is $10^{-2} \mathrm{~m}$ )

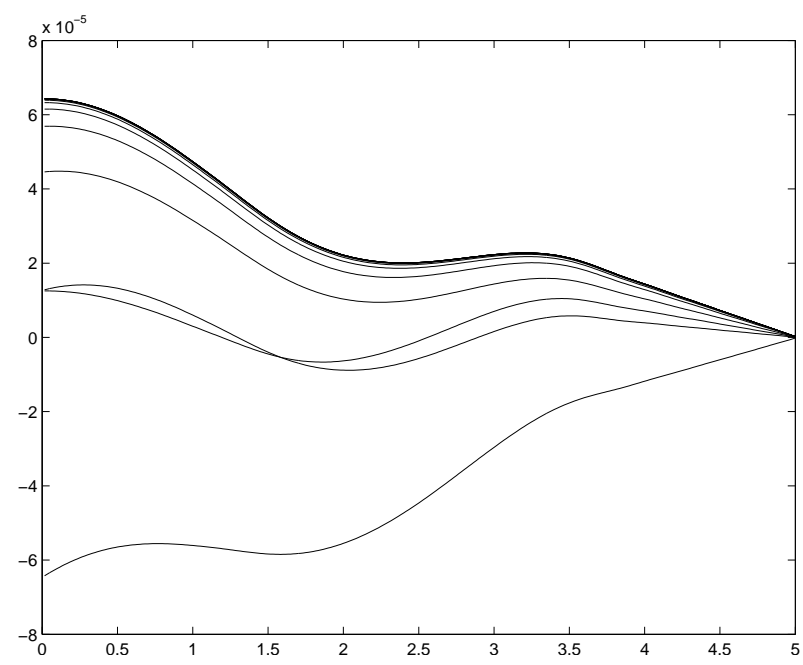

Fig. 4.3. Functions $\psi_{1}\left(t, ., \xi_{l}\right) l=1: L$ at time $t=1.3 \mathrm{~ms}$ 
4.5. Comparison between experimental and theoretical stability conditions. In the case of explicit schemes, the (sufficient) stability conditions are:

condition 1. $\Delta t$ small enough,

condition 2. $a_{l i}-\frac{b_{l i}}{2 \Delta x} \sum_{k} b_{k j}>0$.

We propose to compare from numerical simulations the stability condition 2 with the experimental one. In the same conditions as previously, for different values of $\frac{\Delta t}{\Delta x}$, we test the experimental stability of the schemes and verify if condition 2 is satisfied or not. The results are presented in table 4.1 (resp. in table 4.2) for the first (resp. the second) scheme. In the two cases, the results confirm that condition 2 is a sufficient stability condition. Because the interval of $\frac{\Delta t}{\Delta x}$ values for which condition 2 is not verified whereas the scheme remains stable is small, this condition is in fact "almost necessary".

Finally, to make the link with section 4.3 , we can remark that the experimental stability bounds are intimately linked to propagation velocities. Indeed, the values of velocities defined in section 4.3 are (in length unit $e$ per second):

$$
c=5992, c_{d}=5038 \text { and } v_{d}=6856,
$$

which correspond to the physical values $c=299.6 \mathrm{~m} . \mathrm{s}^{-1}, c_{d}=251.9 \mathrm{~m} . \mathrm{s}^{-1}$ and $v_{d}=342.8 \mathrm{~m} . \mathrm{s}^{-1}$. We can remark that, as expected, the schemes become unstable when $\frac{\Delta x}{\Delta t} \leqslant \frac{1}{1.9810^{-4}} \simeq c_{d}$, that is when the numerical propagation velocity is less than the model's one.

TABLE 4.1

First explicit scheme

\begin{tabular}{|c|c|c|}
\hline value of $\Delta t / \Delta x$ & condition 2 & Stability \\
\hline$\leqslant 1.4710^{-4}$ & verified & yes \\
\hline from $1.4810^{-4}$ to $1.9710^{-4}$ & not verified & yes \\
\hline$\geqslant 1.9810^{-4}$ & not verified & no \\
\hline \hline value of $\Delta t / \Delta x$ & condition 2 & Stability \\
\hline$\leqslant 1.4710^{-4}$ & verified & yes \\
\hline from $1.4810^{-4}$ to $1.9810^{-4}$ & not verified & yes \\
\hline$\geqslant 1.9910^{-4}$ & not verified & no \\
\hline \multicolumn{2}{|c}{ TABLE 4.2} \\
Second explicit scheme
\end{tabular}

Appendix A. A particular time discretization.

For a linear differential system in $\mathbb{C}^{M}$ :

$$
\partial_{t} \varphi=A \varphi+B w, \varphi(0)=0,
$$

the solution $\varphi$ is given by:

$$
\varphi(t)=\int_{0}^{t} e^{A(t-s)} B w(s) d s .
$$

For $w$ constant in $[t-\Delta t, t+\Delta t]$, we have:

$\varphi(t+\Delta t)=\int_{0}^{t-\Delta t} e^{A(t+\Delta t-s)} B w(s) d s+\int_{t-\Delta t}^{t+\Delta t} e^{A(t+\Delta t-s)} d s B w(t)=F \varphi(t-\Delta t)+G w(t)$, 
with $F=e^{2 \Delta t A}$ and $G=A^{-1}\left(e^{2 \Delta t A}-I\right) B$. So we get the following numerical scheme:

$$
\varphi^{t+\Delta t}=F \varphi^{t-\Delta t}+G w^{t} .
$$

Note that this scheme is especially useful in the case where $A$ is diagonal.

\section{REFERENCES}

[1] J. Audounet, F.A. Devy-Vareta, G. Montseny, Pseudo-invariant diffusive control, 14th International Symposium of Mathematical Theory of Networks and Systems (MTNS'2000), Perpignan (France), June 19-23, 2000.

[2] J. Audounet, V. Giovangigli, J.-M. Roquejoffre, A threshold phenomenon in the propagation of a point source initiated flame, Physica D, 1998.

[3] P. Bidan, T. Lebey, G. Montseny, C. Neacsu, J. Saint-Michel, Transient voltage distribution in motor windings fed by inverter: experimental study and modeling, IEEE Trans. on Power Electronics, Vol 16, No 1, Jan. 2001.

[4] B. Cockburn, G. Karniadakis, and C. Shu, Discontinuous Galerkin Methods. Theory, Computation and Applications, Lect. Notes Comput. Sci. Eng. 11, Springer, Berlin, 2000.

[5] S. GASSER, Etude des propriétés acoustiques et mécaniques d'un matériau métallique poreux modèle à base de sphères creuses de nickel, $\mathrm{PhD}$ thesis, Institut National Polytechnique de Grenoble, 2003.

[6] J.-P. Groby, C. TsogkA, A time domain method for modeling wave propagation phenomena in viscoacoustic media, Six International Conference on Mathematical and Numerical aspects of Wave Propagation (Waves 2003), Jyväskylä, June 30-July 4, Finland, 2003.

[7] B. Henry, S. Wearne, Existence of turing instabilities in a two-species fractional reactiondiffusion system, SIAM J. Appl. Math., Vol. 62 No. 3, pp 870-887, 2002.

[8] D. LAfARge, Propagation du son dans les matériaux poreux à structure rigide saturés par un fluide viscothermique, $\mathrm{PhD}$ thesis, Université du Maine, 1993.

[9] L. Laudebat, P. Bidan, G. Montseny, Modeling and Optimal Identification of Pseudodifferential Electrical Dynamics by Means of Diffusive Representation - Part I : modeling, IEEE Trans. on Circ. \& Syst. I, Vol. 51, No. 9, 2004.

[10] T.A.M. LAnglands, B.I. Henry, The accuracy and stability of an implicit solution method for the fractional diffusion equation, Journal of Computational Physics, Vol. 205, Issue 2, pp 719-73620, 2005.

[11] M. Lenczner, G. Montseny, Diffusive realization of operators solutions of certain operational partial differential equations, Comptes Rendus de l'Académie des Sciences (Mathématiques), Vol. 341 - No 12 - pp 737-740, 2005.

[12] D. Levadoux, B. Michielsen, Analysis of a boundary integral equation for high-frequency Helmoltz problems, SIAM, 1998.

[13] A. Lorenzi, F. Messina, Identification problems for Maxwell integro-differential equations related to media with cylindric symmetries, Journal of Inverse and Ill-Posed Problems, Vol. 11, N. 4, pp 411-437, 2003.

[14] G. Montseny, Représentation diffusive, Hermes Science, Paris, 2005.

[15] G. Montseny, Diffusive representation for operators involving delays, "Applications of timedelay systems" (J.-J. Loiseau \& J. Chiasson eds.), pp. 217-232, Springer-Verlag, 2007.

[16] S. R. Pride, F.D. Morgan and A.F.Gangi, Drag forces of porous-medium acoustics, Phys. Rev. B. 47 (1993), 4964-4978.

[17] A. Taflove and S.C. Hagness, Computational Electrodynamics: The Finite-Difference TimeDomain Method, 2nd edition, Artech, Norwood, 2000.

[18] C. M. Topaz and A. L. Bertozzi, Swarming Patterns in a Two-Dimensional Kinematic Model for Biological Groups, SIAM J. Appl. Math., Vol. 65, No 1, pp 152-174, 2004.

[19] K. YosidA, Functional analysis, Springer, 1965. 